\title{
Propuesta para el \\ fortalecimiento de la educación básica (PFEB) en México
}

A Proposal to Strengthen Basic Education in Mexico

\author{
Emiliano López Gutiérrez \\ elgmilo@hotmail.com \\ Costa Rica \\ Lirio Cristell Salgado Molina \\ critell82@hotmail.com \\ Costa Rica \\ Ileana Arauz Beita \\ ileana.arauz.beita@una.cr \\ Costa Rica
}

\section{Resumen}

Se ofrece una propuesta para el fortalecimiento de la educación básica en México. Se analiza la condición de los planteles educativos y si contribuyen a materializar los esfuerzos de la política educativa estatal.

Se comparan los contextos con altos niveles de marginación y pobreza con otros que carecen de este fenómeno. Se plantea que la educación para México es significativa en todos los aspectos, dada la pluralidad y diversidad cultural que ostenta. Se espera incidir de una manera innovadora en la educación de México. Se propone iniciar en los estados con mayor índice de rezago escolar. Se plantea que esta PFEB se aplique primero en el Estado de Guerrero, por las características que presenta este estado en materia de rezago educativo en relación con otros estados de la república mexicana, La metodología utilizada en esta investigación partió de un estudio observacional-documental. Se proponen cambios tanto curriculares, como pedagógicos y de recursos, tanto tecnológicos como de infraestructura. 
En la aplicación de la propuesta, se incluyen las supervisiones o monitoreos de las autoridades competentes.

Palabras clave: diagnóstico; educación general básica; estado de Guerrero fortalecimiento; rezago escolar.

\begin{abstract}
This article offers a proposal to strengthen basic education (PFEB, for its acronym in Spanish) in Mexico. The article analyze the condition of schools and whether they contribute to materialize the efforts of the State education policy. It also compares the contexts with high levels of poverty and marginalization with other contexts lacking of these conditions. It is stated that the education for Mexico is significant in all aspects, given its plurality and cultural diversity. It is expected to have an impact in the Mexican education in an innovative way, starting in the states with the highest rate of educational lag. This PFEB would be implemented first in the state of Guerrero due to the characteristics of this state in the field of educational lag in relation to other states of the Mexican Republic. The methodology used in this research adopted an observational-documentary study design. Curricular, pedagogical, and technological changes are proposed; as well as changes of resources and infrastructure. In the implementation of the proposal, the supervision or monitoring by the competent authorities is included.
\end{abstract}

Keywords: basic general education; educational lag; diagnosis; state of Guerrero; strengthening.

La educación en México ha evolucionado paulatinamente; sin embargo, las sociedades en general muestran una complejidad marcada en cuanto al sector educativo por Estado, contexto donde se generan puntos clave para ser abordados. Básicamente no es posible hegemonizar un esquema de trabajo conjunto, en lugares de altos niveles de marginación y pobreza con otros que no carezcan de este fenómeno; además, los sistemas de educación requieren bases de conocimientos sólidas para los sujetos involucrados en ella, principalmente estudiantes y docentes.

Las políticas educativas apuntan a integrar a la sociedad con las tendencias cambiantes del mundo; no obstante, parte de los cambios deberían ser evaluados a conciencia, una situación por demás relevante, dadas las evidencias contrastantes en el sistema educativo nacional, conformado por estudiantado y personal educativo; autoridades educativas; planes y programas, métodos y materiales educativos; instituciones educativas del Estado y de sus organismos descentralizados; 
instituciones particulares con autorización o con reconocimiento de validez oficial de estudios; instituciones de educación superior a las que la ley otorga autonomía. Este sistema debe ser evaluado confrontado con la realidad de las presentes problemáticas sociales por las que atraviesa el país, pues la consideración del desarrollo humano (entendido como un proceso de aprendizaje y de aplicación de lo aprendido para mejorar la calidad de vida) incide como eje de todo proceso de crecimiento y la necesaria vinculación entre desarrollo y educación (Cruz, 2009).

En este orden de ideas, queda por preguntarse qué tanto pueden haberse alcanzado objetivos importantes en el sector educativo, como el uso de las tecnologías en las escuelas como facilitadoras para la recopilación de información para el alumnado, por no dejar de lado que también acontecen efectos negativos que inciden directamente, específicamente la falta de interés para analizar, asimilar y utilizar el conocimiento adquirido en su formación académica y desarrollo personal. Estas situaciones trasgreden y permiten que la educación carezca de la calidad deseada.

Es un hecho que la educación es significativa para México en todos los aspectos, dada la pluralidad y diversidad cultural que ostenta, por ejemplo, la Organización para la Cooperación y el Desarrollo Económico señala que México ha sido considerado muchas veces como una economía que se encuentra en una fase de "nivelación", en donde el énfasis para mejorar la difusión y la calidad del capital humano es crucial para el futuro desarrollo económico y social (OECD, 2004). Los retos sociales y culturales para este país son igualmente importantes e incluyen la consolidación de una democracia moderna y de una sociedad multicultural con igualdad de oportunidades para toda la población, en fin, humanista.

La Propuesta Fortalecimiento de la Educación Básica (PFEB) en su objetivo general incidir de manera directa en el proceso de enseñanza-aprendizaje como una herramienta integradora, que agrupe nuevamente las responsabilidades conjuntas de los planteles educativos y de cada integrante.

Esta propuesta PFEB podría iniciar su aplicación en el estado de Guerrero, por las características que presenta este estado en materia de rezago educativo en comparación con otros de la República Mexicana. A partir de esta aplicación y, si la propuesta manifestara una alternativa viable para reforzar este nivel educativo, podría adquirir características de un modelo educativo de calidad incluyente, pues permitiría incluir aquellas situaciones del plantel educativo y no se han 
considerado en ningún otro modelo educativo ya implementado. Entonces, al iniciar la aplicación podría existir la opción de que la propuesta pueda fomentar cambios correctivos, con una proyección a futuro para incorporarse a nivel nacional en beneficio de la calidad educativa y, de esta forma, contribuir de manera positiva a la sociedad mexicana.

La metodología utilizada en esta investigación partió de un estudio observacional-documental.

El PFEB está dirigido completamente al sector educativo de nivel básico, el cual contempla como premisa monitoreos constantes, a partir de la visita o supervisión de cada plantel (Schoolar Mistery Guest), pero con fines específicos, como asegurar que el proceso de enseñanza-aprendizaje se esté realizando debidamente; además, se identifican las debilidades que no se plasman dentro del plan de mejora o en las reuniones de consejo técnico que normalmente se llevan a cabo en las instituciones educativas. Con ello, también se analizan las prácticas que que no favorecen la educación como: la corrupción, la complicidad en el proceso educativo a través de prácticas repetitivas negativas, la falta de innovación y corrección de errores, el fomento de prácticas irregulares y la simulación. Además, se puede identificar si se presentan faltas de respeto entre el personal y sus estudiantes, como indicador de alto impacto en el desarrollo y formación del alumnado y si se refuerzan de manera especializada los valores, cívica, ética, entre otros.

La presente propuesta busca incidir de una manera innovadora en la forma en la que se ha desarrollado le educación en México, a partir de un análisis que inicia en uno de sus estados con mayor índice de rezago escolar. Actualmente existen condiciones para desarrollar nuevas estrategias que apoyen esta propuesta en beneficio de la sociedad, debido a que la reforma educativa establece cambios orientados a establecer como objetivo la búsqueda de la calidad educativa, reconocer la identidad cultural de nuestro país, garantizar la igualdad de todos al disfrute de este derecho y la rectoría de la Federación en cuanto a la creación de los planes y programas para los niveles preescolar, primaria, secundaria y normal (SEP 2, 2013).La dinámica actual del sistema educativo y las características generales del Estado presentan un panorama de desafíos y debilidades. El grado promedio de escolaridad de la población mayor de quince años, en el año 2000, fue de 6,1 grados, el cual se logró modificar a 7,3 grados en el 2011, con un avance de 1,2 grados que ubican la entidad en el antepenúltimo 19 Programa Sectorial 2011-2015 de Educación. Por su parte, los promedios nacionales de este 
indicador se establecieron en 7,5 y 8,6 respectivamente. El rezago educativo en Guerrero es de un 1206386 habitantes mayores de 15 años, en el que se incluye la población analfabeta, la que no concluyó la educación básica, que representa, según datos del INEGI 2010, el 53,8 \% del total de la población de este rango de edad. Esto significa que uno de cada dos guerrerenses se encuentra en condición de rezago. Existe el reto de cobertura en educación básica, principalmente en preescolar, secundaria medio rural, así como, en educación indígena, evidenciado en nuestros desfavorables indicadores como muestra del rezago educativo. Al sistema no escolarizado correspondió el 8,4\% de nuestra población escolar, el que atiende a 101109 personas usuarias.

El 82,2 \% de la población escolar se ubica en el nivel básico, que comprende la educación preescolar, primaria y secundaria. La educación media superior representa el 9,8\% de la matrícula y constituye el tipo educativo que proyecta el mayor crecimiento, resultado de la expansión de la educación básica en los próximos años, así como por la obligatoriedad recientemente puesta en marcha para este nivel; en cuanto a la educación superior, con 64088 estudiantes que representan el 5,8\% de la matrícula total y el 1,6\% restante corresponde a los servicios de capacitación para el trabajo

En la educación primaria asistieron al ciclo escolar 2010-2011, 520680 niños, niñas y jóvenes de 6 a 14 años de edad, equivalente al 57,7 \% de la educación básica y $47,5 \%$ de todo el sistema educativo escolarizado, lo que permite ubicar a este nivel educativo como el de mayor dimensión entre la población escolar. En relación con la cobertura, el Estado registra el 124,9 \% mientras que la media nacional es del $117,7 \%$, situación que se genera por la atención de alumnado en rezago, extra edad, capacidades diferentes y movilidad migratoria, entre otras causas.

Es importante señalar que el estado de Guerrero enfrenta aún el reto de oferta educativa para este nivel, ya que gran cantidad de niñez en edad escolar no asiste a la escuela por diversas circunstancias, principalmente marginación, pobreza y falta de servicios en localidades apartadas, lo que contrasta nuestro indicador de cobertura que es de $124,9 \%$, pues prácticamente atendemos gran parte del rezago que incide en alumnado extra edad.

En el proceso educativo, algunos factores que permean en el nivel de secundaria, son la inseguridad, las adicciones, inmadurez de identidad por el tránsito a la adolescencia, violencia intrafamiliar, embarazo precoz y la desintegración 
familiar, la contribución económica que jóvenes realizan para el sustento familiar, poco interés de algunos padres y madres sobre la continuidad de los estudios de sus hijos e hijas, falta de compromiso compartido en las tareas escolares y la reprobación de asignaturas que impactan en los indicadores educativos, así como formación académica distinta a la pedagógica de parte de docentes, resistencia a los procesos de actualización. Otra parte carece de vocación y compromiso para el desempeño de la función encomendada. No se ha consolidado, entre comunidad educativa y sociedad, la concepción de educación básica para la vida, ni se ha logrado mejorar el ambiente formativo para que los grupos de adolescentes alcancen el perfil de egreso adecuado.

La Reforma de Educación Básica es una de las políticas estratégicas educativas que plantea retos, que requieren revisar nuestras prácticas educativas orientadas al cumplimiento de las prioridades del nivel. En este sentido, se trazan rutas viables que permitan gradualmente implementar el nuevo plan de estudios (Tabla 1). Se plantean acciones de difusión efectiva para todos los actores implicados e interesados en este nivel educativo. También se prevén acciones de seguimiento y asesoría a los maestros y maestras, con el fin de garantizar la consolidación de la Reforma Integral de la Educación Básica (SEP, 2011).

Tabla 1

Indicadores básicos educación secundaria 2005-2006 VS 2010-2011.

\begin{tabular}{llllllll}
\hline Descripción & $\mathbf{2 0 0 5 - 2 0 0 6}$ & & & $\mathbf{2 0 1 0 - 2 0 1 1}$ & $\begin{array}{l}\text { Variación } \\
\text { Estatal }\end{array}$ \\
\hline & Estatal & Nacional & Posición & Estatal & Nacional & Posición & \\
Absorción & 88,4 & 94,09 & 31 & 91,5 & 96,5 & 31 & 3,1 \\
Deserción & 8,09 & 07,07 & 26 & 6,06 & 5,06 & 26 & 2,3 \\
Reprobación & 15,7 & 18,00 & 11 & 13,3 & 15,9 & 09 & 2,4 \\
Eficiencia terminal & 73,6 & 78,02 & 31 & 78,9 & 83,3 & 29 & 5,3 \\
\hline
\end{tabular}

Nota: Reporte de Indicadores Educativos SEP, 2010.

Los elementos que integran la propuesta incluyen las siguientes acciones:

Supervisar los planteles educativos como el Schoolar Mistery Guest, una iniciativa innovadora para el diagnóstico del proceso de enseñanza - aprendizaje realizado en planteles de educación básica. 
Detectar si se realizan actividades artísticas como danza, flauta, dibujo, dibujo, obras teatrales, manualidades, pintura, entre otros.

Determinar si se realizan talleres para incentivar la lectura y redacción.

Verificar si se presentan actividades que apoyen la niñez que requiera atención por alguna discapacidad o trastorno, como hiperactividad, o una situación especial como la desintegración familiar $\mathrm{u}$ otras como temas específicos que refieren a problemas educativos relevantes en el ámbito social, coherentes con las temáticas apuntadas y que quienes investigan consideran propios de la teoría de la educación.

Determinar tipos de apoyos en: educación moral, educación para la ciudadanía, educación ambiental, educación y desarrollo sostenible, educación para la salud, prevención de la drogadicción, valores y educación, enseñanza de actitudes y valores, las normas y la intervención educativa, estilos cognitivos e intervención pedagógica, educación intercultural, enseñanza de estrategias de aprendizaje, el autoconcepto y su mejora, comunicación y educación, entre muchas otras (Gargallo, 2003).

Por otra parte, es indispensable inferir que en la actualidad, por medio de las TIC y redes sociales, el alumnado ha adquirido una herramienta que le permite interactuar y estar informado en mayor proporción que generaciones anteriores. Por este motivo, el PFEB pretende, primeramente, diagnosticar cómo se está realizando el arte de la educación en los planteles, para evaluar cuáles podrían ser las estrategias adecuadas para redirigir las políticas en materia de educación y, en este sentido, preparar al alumnado de educación básica hacia el siguiente nivel, como un medio o forma de transición al nivel medio superior del sistema educativo nacional, considerando las actualizaciones del sistema educativo, como las escuelas de tiempo completo o de horario normal. Por ejemplo, el Plan Nacional de Desarrollo plantea que una educación de calidad demanda congruencia de la estructura, organización y gestión de los programas educativos, con la naturaleza de los contenidos de aprendizaje, procesos de enseñanza y recursos pedagógicos, para que se atienda con eficacia el desarrollo de las capacidades y habilidades individuales en los ámbitos intelectual, artístico, afectivo, social y deportivo, al mismo tiempo que se impulsa una formación en valores favorable a la convivencia solidaria y comprometida, que prepare individuos que ejerzan una ciudadanía activa, capaces de enfrentar la competitividad y exigencias del mundo del trabajo. El Programa Nacional de Educación 2001-2006 señala que la educación básica 
-preescolar, primaria y secundaria- es la etapa de formación de las personas en la que se desarrollan las habilidades de pensamiento y las competencias básicas para favorecer el aprendizaje sistemático y continuo, así como las disposiciones y actitudes que normarán su vida (SEP, 2006).

Finalmente, en una siguiente etapa, la propuesta debe estar encaminada a incorporar profesionales o personal investigador altamente calificado como las nuevas generaciones egresadas del Consejo Nacional de Ciencia y Tecnología (CONACYT), para integrar a nuevos maestros y maestras con reconocimiento nacional en esta dinámica, con el propósito de asegurar una educación de calidad. En este sentido, se permea la generación de empleos, es decir, la propuesta es incluyente, debido a que contempla la participación de personal profesional certificado a nivel nacional. Además, existiría la viabilidad necesaria para instrumentar un sistema educativo mexicano con mayor solidez y así erradicar las malas prácticas o simulación en el sistema educativo nacional.

Los esfuerzos particulares son loables; sin embargo, no es posible dejar en mano de personajes espontáneos que utilizan programas públicos de dependencias de ayuda social para obtener recursos económicos en favor de actividades educativas que no son supervisadas. Resulta perjudicial la inexistencia de controles adecuados que aseguren que dichas actividades son aplicables al contexto del plantel o si el personal que las imparte está calificado para impactar positivamente en el alumnado.

Al existir candados o credenciales de rigor académico, los planteles tendrían una herramienta importante para identificar que los apoyos externos sean de calidad para el alumnado. De esta manera, existe la posibilidad de crear las sinergias para mejorar la calidad de la educación en México. Por ejemplo, en este nivel se podría incluir la orientación educativa de manera permanente, por medio de programas externos como los emitidos por SEDESOL, pero contemplando los lineamientos del PFEB, el cual propone que todo personal que se vincule directamente con estudiantes esté altamente calificado y esté capacitado para considerar la realidad del contexto social donde labora.

La presente propuesta toma en consideración realidades existentes, que han afectado e impactado de manera seria el estado de Guerrero en materia educativa. Por lo tanto, es imperativa la participación activa del personal investigador que conoce la forma en la cual se desarrolla la educación en este estado, como conocedor del porqué y las estrategias por seguir para ayudar positivamente a la formación 
de nuevas generaciones con la inteligencia necesaria y temprana que les permita visualizar un contexto de vida sano, a través de las mejores decisiones y de carácter personal.

Se espera que la educación realmente sea significativa para cada estudiante, porque es un hecho que el personal docente, por vocación, cuenta con el conocimiento necesario para lograr que los aprendizajes contribuyan a fortalecer la educación en el Estado. Por ello, como parte de la propuesta, es necesario identificar si el personal docente actual logra cumplir los objetivos nacionales en el tema de educación y si podrían apoyar la propuesta. Su calidad profesional, desempeño laboral, compromiso con los resultados, etc., son algunas de las preocupaciones centrales del debate educativo orientado a la exploración de algunas claves para lograr que la educación responda a las demandas de la sociedad actual, en armonía con las expectativas de las comunidades, familias y estudiantes (Machado, 2006).

En la educación no puede existir lugar a duda, debe imperar el compromiso conjunto para mejorar la condición de vida de las personas, pues la educación sigue ocupando un lugar central para el desarrollo social del país, aunado a otros factores prioritarios que obligan al gobierno y a la sociedad a poner mayor énfasis en la formación integral de las nuevas generaciones (SEP, 2003).

El desafío es fortalecer la educación. Para tal efecto, se requiere la participación de profesionales o personal investigador con vocación para generar un cambio positivo en favor de nuestro país, México. El reto es de grandes dimensiones y los cimientos para llevar a cabo los cambios siempre han sido los individuos: personas mejor formadas, ciudadanía respetuosa de las leyes y de su entorno, seres humanos creativos y solidarios, sujetos que comprenden y aprenden, capaces de enfrentar desafíos, superar adversidades y construir un mejor mañana (SEP, 2013).

\section{Referencias}

Cruz, I. (2009). Breve evolución de los sistemas educativos latinoamericanos: Necesidad de la educación para el desarrollo sostenible. Revista Iberoamricana de Educación, 49(7).

Gargallo López, B. (2009). La Teoría de la Educación. Objeto, enfoques y contenidos. Revista Interuniversitaria, 14. Recuperado de: http://revistas.usal.es/ index.php/1130-3743/article/view/2967 
Machado, A. L. (2006). Modelos innovadores en la formación inicial docente en América Latina y Europa. Santiago de Chile: Oficina Regional de Educación de la UNESCO para América.

La Organización para la Cooperación y el Desarrollo Económico (OECD). (2004) Revisión nacional de investigación y desarrollo educativo. México. Reporte de los examinadores sobre México.

Secretaría de Educación Básica (SEP). (2006). Acuerdo número 384. México: Diario Oficial.

Secretaría de Educación Básica (SEP). (2011). Programa sectorial de educación 2011-2015. Diagnóstico del sistema educativo estatal. Guerrero, México

Secretaría de Educación Básica (SEP). (2013). Programa Sectorial de Educación 2013-2018. México, D. F. Primera Edición.

Secretaría de Educación Básica (SEP). (2013). Reforma Educativa 2013. México: Diario Oficial de la Federación.

Subsecretaría de Educación Básica (SEP). (2003). Plan estratégico. Guerrero, Chilpancingo. 